\title{
Treating Opioid Addiction With Buprenorphine-Naloxone in Community- Based Primary Care Settings
}

Ira L. Mintzer, $M D^{1}$

Mark Eisenberg, $M D^{2}$

Maria Terra, $B A^{1}$

Casey MacVane, MD, MPH ${ }^{2}$

David U. Himmelstein, $M D^{1}$

Steffie Woolhandler, $M D^{1}$

'Harvard Medical School/Cambridge Health Alliance, Cambridge, Mass

${ }^{2} \mathrm{MGH}$-Charlestown HealthCare Center/ Harvard Medical School, Boston, Mass

Conflicts of interest: none reported

\section{CORRESPONDING AUTHOR}

Ira L. Mintzer, MD

The Cambridge Hospital

1493 Cambridge St

Cambridge, MA 02139

imintzer@challiance.org

\begin{abstract}
PURPOSE Office-based treatment of opioid addiction with a combination of buprenorphine and naloxone was approved in 2002. Efficacy of this treatment in nonresearch clinical settings has not been studied. We examined the efficacy and practicality of buprenorphine-naloxone treatment in primary care settings.

METHODS We studied a cohort of 99 consecutive patients enrolled in buprenorphine-naloxone treatment for opioid dependence at 2 urban primary care practices: a hospital-based primary care clinic, and a primary care practice in a free-standing neighborhood health center. The primary outcome measure was sobriety at 6 months as judged by the treating physician based on periodic urine drug tests, as well as frequent physical examinations and questioning of the patients about substance use.
\end{abstract}

RESULTS Fifty-four percent of patients were sober at 6 months. There was no significant correlation between sobriety and site of care, drug of choice, neighborhood poverty level, or dose of buprenorphine-naloxone. Sobriety was correlated with private insurance status, older age, length of treatment, and attending selfhelp meetings.

CONCLUSIONS Opioid-addicted patients can be safely and effectively treated in nonresearch primary care settings with limited on-site resources. Our findings suggest that greater numbers of patients should have access to buprenorphinenaloxone treatment in nonspecialized settings.

Ann Fam Med 2007;5:146-150. DOI: 10.1370/afm.665.

\section{INTRODUCTION}

T n October 2002 the US Food and Drug Administration approved buprenorphine (a unique $\mu$-receptor partial agonist) for the treatment of opioid dependence. ${ }^{1}$ To minimize the potential for abuse and diversion, buprenorphine is often compounded with a small quantity of naloxone. Until June 2005, federal regulations limited the number of concurrent patients to 30 who could be treated with buprenorphine-naloxone at any single institution. Presently, each prescribing physician is allowed to treat 100 patients. Physicians are required to undergo a special 8-hour training in buprenorphine-naloxone therapy or to be a certified addiction specialist to prescribe this medication in an outpatient office.

Opiate-agonist pharmacotherapy has been shown to decrease illicit opiate use. ${ }^{2} \mathrm{~A}$ randomized controlled trial established the safety and efficacy of a sublingual buprenorphine-naloxone preparation in the treatment of opiate addiction. ${ }^{3}$ Patients in that study were seen on a weekly basis with medication administered from an on-site pharmacy. Take-home doses were dispensed for weekends and holidays. All patients received individual counseling from an on-site counselor and were encouraged to receive behavioral treatment services apart from the study. A systematic review of randomized 
controlled trials found that buprenorphine was superior to placebo, but not to methadone. ${ }^{4}$

Few studies have documented the outcomes of buprenorphine-naloxone treatment outside such resource-rich research settings. We examined the experience of 2 office-based practices in providing induction, stabilization, and maintenance doses of buprenorphine-naloxone. Patients received their care in 2 primary care clinics serving low- and middle-income urban neighborhoods: a hospital-based primary care center, and a neighborhood health center.

\section{METHODS}

\section{Treatment Protocols}

The treating physicians were general internists in busy urban practices devoted to primary care. Patient visits occurred during normal primary care sessions. One physician was a certified addiction specialist; the other 2 received 8 hours of training in buprenorphine-naloxone treatment as required by law.

At both sites, patients were either self-referred or referred by practitioners in affiliated practices. In both cases, no special outreach efforts were required; awareness of the availability of buprenorphine-naloxone treatment spread quickly by word of mouth among colleagues and community members, resulting in demand for treatment that exceeded the legal limit of 30 patients per site.

All patients were strongly encouraged (but not required) to attend meetings of Alcoholics Anonymous (AA) or Narcotics Anonymous (NA). Both sites used buprenorphine-naloxone to treat all patients (hereafter referred to as buprenorphine.)

The treatment protocols at the 2 sites differed slightly because of resource constraints. The hospitalbased primary care center had access to a pharmacy but did not have an on-site addictions counselor. Conversely, the neighborhood health center had a counselor, but not a pharmacy, at the site.

At the hospital-based primary care center, patients were seen by a physician for an initial evaluation, and then scheduled for visits on 3 consecutive days for the induction of buprenorphine therapy. Patients were instructed to abstain from opiate use for 24 hours before the first induction visit with the expectation that they would be in mild withdrawal. At that induction visit, doses of buprenorphine obtained from the on-site pharmacy were administered over 4 hours in 2 divided doses (maximum dose $8 \mathrm{mg}$.) On day 2, the efficacy of the first day's dosing was evaluated, and a dose of up to $24 \mathrm{mg}$ of buprenorphine was administered sublingually. On day 3, patients received a stabilization dosage of buprenorphine, ranging from 8 to $24 \mathrm{mg}$ a day.
At that time patients received a prescription for 1 to 2 weeks of buprenorphine, and they were asked to return in 1 to 2 weeks for further evaluation. All patients were asked to visit an outpatient addiction center at a separate location for intake and group therapy. Patients were seen monthly after stabilization.

At the neighborhood health center, patients were seen by 1 of 2 physicians. Patients were also evaluated by an on-site addictions counselor. On day 1 patients were given a prescription for buprenorphine, which they were instructed to self-administer when they were in active withdrawal. They were subsequently reevaluated on days $3,7,14$, and 21 . After achieving a stabilization dosage, they were seen during monthly follow-up visits. Patients generally received stabilization dosages ranging from 8 to $24 \mathrm{mg}$ a day. All patients were advised to attend AA or NA meetings and were referred for community mental health evaluation.

\section{Analytic Methods}

Consecutive patients receiving buprenorphine therapy at either of the 2 sites were enrolled in the study. Four patients who initially sought buprenorphine treatment at the neighborhood health center site but failed to return for a second visit were excluded from analysis.

Chart data was abstracted by 2 authors (M.T. and C.M.) using a data form developed by the treating clinicians involved in the study (I.L.M. and M.E.).

Our primary outcome variable was sobriety at 6 months. Sobriety was determined from the global assessment of the treating physician. In all cases, the physician questioned the patient on each visit about possible substance use and performed a brief physical examination. In addition, the physician ascertained attendance at AA or NA meetings and counseling sessions, and reviewed adherence to buprenorphine dosing. At the hospital-based clinic, intermittent urine drug tests were obtained to confirm sobriety during the first several months of the study. Subsequently, urine drug tests were obtained at every patient visit. At the neighborhood health center, urine drug tests were obtained at every visit throughout the study. Patients with a single positive drug test or other evidence of brief relapse were not automatically dismissed from the treatment programs. In such cases, motivated patients were sometimes offered intensified counseling and/or adjustment of buprenorphine dosage. Repeated or prolonged relapse resulted in dismissal.

In one case a patient moved away after 4 months of treatment for reasons clearly unrelated to drug relapse (being called up for military service in Iraq). In that case, sobriety was determined by global clinical assess- 
ment at the last follow-up visit. All other patients who were dismissed or lost to follow-up were assumed to have relapsed in their substance abuse.

Demographic variables obtained from patients' charts included age, sex, and race. We assessed 2 surrogate measures of socioeconomic status (SES): (1) poverty rate within the census tract where the patient resides, and (2) type of health insurance. Data on census tract poverty rate was obtained from the Census Bureau through the Federal Financial Institutions Examination Council's Geocoding System. ${ }^{5}$ We initially classified health insurance as (1) private insurance, (2) self-pay, or (3) Free Care Pool/Medicaid. In Massachusetts, where the study was conducted, Free Care Pool refers to a specific insurance-like reimbursement mechanism for low-income patients. Interpretation of the insurance variable is complex, since type of insurance predicts both the patient's out-of-pocket costs for buprenorphine treatment and the patient's SES. To examine the effects of SES (rather than reimbursement-related issues per se), we also analyzed insurance status dichotomously, as private insurance vs self-pay/Medicaid/Free Care Pool, based on the assumption that private insurance correlates with higher SES.

Clinical variables included the patients' principle drugs of abuse (heroin, illicit methadone, or oxycodone/Oxycontin) and hepatitis $\mathrm{C}$ serologic status. Treatment-related variables included site of care (hospital-based primary care clinic vs neighborhood health center), duration of treatment in days, dosage of buprenorphine, and attendance at meetings sponsored by AA or NA.

We also obtained qualitative data on staff reactions to participation in buprenorphine treatment programs. The physicians queried nursing personnel and receptionists regarding (1) disruptive behaviors by patients in the waiting rooms or the clinic environs, and (2) staff willingness to continue treating such patients in the future.

Data were analyzed using SAS-PC. ${ }^{6}$ Mean values of all variables were computed in both sober and nonsober patients. Univariate correlation coefficients between each independent variable and sobriety were assessed, and $\chi^{2}$ tests for significance were computed. Multiple logistic regression was then performed, with variables entered into the model only if the variable's univariate correlation with sobriety was statistically significant at the $P<.10$ level. Separate models were used to assess insurance status (as described above) coded as a 3-level variable and dichotomously.

The study was approved by the institutional review boards at the Cambridge Health Alliance and the Massachusetts General Hospital.

\section{RESULTS}

Ninety-nine patients were studied, 45 from the hospital-based primary care center and 54 from the neighborhood health center. Demographic characteristics are displayed in Table 1. Sixty-four percent of patients were male; $94 \%$ were non-Hispanic whites, $2 \%$ were Hispanic, and $3 \%$ were nonHispanic African Americans. The median age was 29 years; no patient was younger than 20 years, and only $10 \%$ were older than 49 years. One quarter of patients were seropositive for hepatitis C. Twentyseven percent of patients had private insurance.

Forty-five percent of patients lived in neighborhoods where the poverty rate was $10 \%$ or less, $37 \%$ lived in neighborhoods with poverty rates of $10 \%$ to $20 \%$, and $18 \%$ lived in neighborhoods with poverty rates of greater than $20 \%$. Forty-seven percent of patients regularly attended meetings of AA or NA.

Overall, $54 \%$ of patients were sober at 6 months: $51 \%$ of those treated at the neighborhood health center and $58 \%$ of those treated at the hospital-based primary care clinic. On univariate analysis (Table 2) the following factors predicted sobriety: private insurance coverage $(P<.05)$, older age $(P<.05)$, and longer duration of treatment $(P<.001)$. Attendance at meetings of AA or NA showed a trend $(P=.09)$ toward positive correlation with sobriety. Self-pay patients were less likely than those with some form of third-party reimbursement (public or private) to maintain sobriety $(P$ $<.03)$. There was no significant correlation between sobriety and site of care, neighborhood poverty level, sex, hepatitis C seropositivity, drug of abuse, or dosage of buprenorphine.

In multivariate models containing all variables found (in univariate analyses) to be significant at the $P<.10$ level, only duration of treatment remained a significant predictor of sobriety $(P=<.001)$, although confidence intervals were large. Because duration of treatment was highly correlated with sobriety (and may be a surrogate measure of sobriety rather than a

\section{Table 1. Selected Characteristics of Patients Receiving Buprenorphine-Naloxone Treatment at 2 Primary Care Sites $(\mathbf{n}=99)$}

\begin{tabular}{lc}
\hline Characteristics & Value \\
\hline Female, \% & 36 \\
Mean age, years & 33 \\
Non-Hispanic white, \% & 94 \\
Seropositive for hepatitis C, \% & 25 \\
Average time in treatment, days & 105 \\
Privately insured, \% & 27 \\
Mean stabilization dose of buprenorphine, mg & 15 \\
\hline
\end{tabular}


Table 2. Characteristics of Patients Treated With BuprenorphineNaloxone Who Did and Did Not Maintain Sobriety Over 6 Months

\begin{tabular}{lccc}
\hline Characteristic & $\begin{array}{c}\text { Sober } \\
\text { Patients } \\
\text { (n = 53) }\end{array}$ & $\begin{array}{c}\text { Nonsober } \\
\text { Patients } \\
\text { (n = 46) }\end{array}$ & P Value \\
\hline Attended AA or NA, \% & 55 & 37 & .09 \\
Private insurance, \% & 36 & 18 & .05 \\
Self-pay, \% & 4 & 18 & .02 \\
Length of time in treatment, days & 169 & 62 & $<.01$ \\
Mean buprenorphine dosage, mg/d & 15.5 & 15.3 & .86 \\
Female, \% & 34 & 40 & .54 \\
Seropositive for hepatitis C, \% & 26 & 24 & .82 \\
Addiction to heroin, \%* & 70 & 78 & .37 \\
Addiction to methadone, \%* & 6 & 0 & .11 \\
Addiction to oxycodone/Oxycontin, \%* & 40 & 38 & .85 \\
Treated at hospital-based primary care site, \% & 49 & 42 & .50 \\
Living in ZIP codes with poverty rates below 10\%, \% & 46 & 43 & .79 \\
Average age, years & 35 & 31 & .05 \\
\hline AA = Alcohol Anonymous; NA = Narcotics Anonymous. & & & \\
* Some patients were addicted to >1 drug. & & & \\
\hline
\end{tabular}

The strength of this study is its enrollment of unselected patients in typical urban practice settings. A weakness is the relatively small number of patients enrolled (a consequence of federal regulations limiting the number of patients who could be treated at each center), which limits the power of our study to assess predictors of successful treatment. Additionally, ascertainment of sobriety may be problematic in some cases. At the outset of the study, the determination of relapse at one clinic site was based on clinical grounds with only periodic drug testing. Only a few patients were enrolled before routine drug testing was implemented, however, and the proportion judged

predictor of it), we repeated the multivariate analysis with duration of treatment excluded and the insurance variable coded dichotomously. In this analysis, private insurance was the only significant predictor of sobriety $(P=.03)$ with a trend toward higher sobriety in patients who attended meetings of AA or NA $(P=.07)$ and were older $(P=.07)$.

\section{Adverse Events}

One sober patient with no history of heart disease died suddenly of previously undiagnosed cardiomyopathy. No drugs were found in his blood or urine. Interviews with staff found no instances of disruptive behaviors among patients undergoing buprenorphine treatment. All staff felt comfortable continuing to work in settings where buprenorphine treatment was administered.

\section{DISCUSSION}

Our findings suggest that opioid-addicted patients can be treated safely in nonresearch, primary care settings with limited on-site resources. The success rates we obtained are similar to those reported from more specialized treatment centers using methadone or LAAM (L-alpha-acetylmethadol) (between 50\% and 60\%).7,8 A few reports have documented good retention rates with buprenorphine. ${ }^{9,10}$ In contrast, our study used sobriety at 6 months rather than mere retention as the outcome measure. Other studies have found low rates of success with short-term detoxification and nonpharmacotherapeutic treatments (5\%-20\%) and with naltrexone-alone maintenance treatment (10\%-20\%). ${ }^{11}$ sober did not change after this revision in protocol. At both sites, urine samples were obtained unwitnessed, and clinician assessment of sobriety is subject to error. Meeting the reference standard of frequent, unscheduled, fully-observed drug testing is impractical in the community setting, however, and judgment of sobriety and social functioning by experienced clinicians combining drug screening and interviews is likely to be reasonably reliable.

The 2 settings included in the study used slightly different treatment protocols because of limitations in on-site resources (no pharmacy at the neighborhood health center and no addictions counselor at the hospital-based primary care center.) Clinicians in most nonresearch settings are likely to face similar limitations. Hence, we believe that our findings are relevant to the real-world practice of addictions treatment.

The single adverse patient event-an unexpected cardiac death—was severe. Because of our small sample size and the absence of an untreated control group, we cannot assess whether buprenorphine treatment caused this death. The baseline risk of death is probably high among narcotic addicts. ${ }^{12,13}$ Nonetheless, continued, careful postmarketing surveillance of buprenorphine (and all other new medications) is clearly warranted.

Somewhat surprisingly, we saw no disruptive behaviors among patients receiving buprenorphine therapy. Although both physicians and their staffs are willing to continue treating addicted patients at their practice site, several aspects of caring for these patients were challenging. Both clinics received a high volume 
of calls from prospective patients inquiring about the possibility of treatment, presumably due to the dearth of physicians offering buprenorphine treatment. The multiple visits required during the induction phase of treatment resulted in scheduling problems, particularly at the hospital-based clinic, where patients were observed on-site for 4 hours after the induction dose of buprenorphine was administered.

Having private insurance correlated with sobriety, whereas residence in a neighborhood with high poverty rates did not. We interpret these somewhat contradictory results as suggesting that employment (which probably correlates highly with private insurance) is a favorable prognostic factor for narcotic addicts, regardless of whether they live in a high-poverty neighborhood. Attending AA or NA meetings and older age were also factors that showed a trend toward predicting treatment success. These results support our clinical impression that group treatment is an important adjunct to recovery.

Our study indicates that opioid-dependent patients can be successfully treated in nonspecialized, primary care settings with limited resources. Our findings lend support to recent effort to loosen regulations ${ }^{14}$ and allow greater numbers of patients access to buprenorphine treatment in nonspecialized settings. ${ }^{15}$ We hope that our findings will encourage other primary care physicians to consider providing this efficacious form of care.

To read or post commentaries in response to this article, see it online at http://www.annfammed.org/cgi/content/full/5/2/146.

Key words: Opiate addiction; narcotic addiction/drug therapy; narcotic antagonists; substance abuse; primary health care; primary care issues; research, practice-based; patient selection

Submitted June 19, 2006; submitted, revised, October 10, 2006; accepted October 23, 2006.

\section{References}

1. Pani PP, Maremmani I, Pirastu R, Tagliamonte A, Gessa GL. Buprenorphine: a controlled clinical trial in the treatment of opioid dependence. Drug Alcohol Depend. 2000;60(1):39-50.

2. Johnson RE, Jaffe JH, Fudala PJ. A controlled trial of buprenorphine treatment for opioid dependence. JAMA. 1992;267(20):2750-2755.

3. Fudala PJ, Bridge TP, Herbert $S$, et al. Office-based treatment of opiate addiction with a sublingual-tablet formulation of buprenorphine and naloxone. N Engl J Med. 2003;349(10):949-958.

4. Mattick RP, Kimber J, Breen C, Davoli M. Buprenorphine maintenance versus placebo or methadone maintenance for opioid dependence. Cochrane Database Syst Rev. 2003(2):CD002207.

5. Federal Financial Institutions Examination Council (FFIEC) Geocoding System. Available at: http://www.ffiec.gov/Geocode/GeocodeSearchmapping.

6. SAS System for Windows. Version 8e. Cary, NC: SAS Institute; 2005.

7. Johnson EE, Christie MJ, Connor M. The role of opioid receptor phosphorylation and trafficking in adaptations to persistent opioid treatment. Neurosignals. 2005;14(6):290-302.

8. O'Connor PG, Oliveto AH, Shi JM, et al. A randomized trial of buprenorphine maintenance for heroin dependence in a primary care clinic for substance users versus a methadone clinic. Am J Med. 1998;105(2):100-105.

9. Stein MD, Cioe P, Friedmann PD. Buprenorphine retention in primary care. J Gen Intern Med. 2005;20(11):1038-1041.

10. Lintzeris N, Bell J, Bammer G, Jolley DJ, Rushworth L. A randomized controlled trial of buprenorphine in the management of short-term ambulatory heroin withdrawal. Addiction. 2002;97(11):1395-1404.

11. Bridge TP, Fudala PJ, Herbert S, Leiderman DB. Safety and health policy considerations related to the use of buprenorphine/naloxone as an office-based treatment for opiate dependence. Drug Alcohol Depend. 2003;70(Suppl 2):S79-S85.

12. Des Jarlais DC, Friedman SR, Sotheran JL, et al. Continuity and change within an HIV epidemic. Injecting drug users in New York City, 1984 through 1992. JAMA. 1994;271(2):121-127.

13. Ghodse H, Oyefeso A, Kilpatrick B. Mortality of drug addicts in the United Kingdom 1967-1993. Int J Epidemiol. 1998;27(3):473-478.

14. Fiellin DA, O'Connor PG. New federal initiatives to enhance the medical treatment of opioid dependence. Ann Intern Med. 2002;137(8):688-692.

15. Fiellin DA, Rosenheck RA, Kosten TR. Office-based treatment for opioid dependence: reaching new patient populations. Am J Psychiatry. 2001;158(8):1200-1204. 